\title{
The Future of Snapchat: A Mathematical Model
}

\author{
Salma M. Al-Tuwairqi*, Bushra K. Aloosh, Rana A. Bagies \\ Mathematics Department, King Abdulaziz University, Jeddah, Saudi Arabia \\ Email: *saltuwairqi@kau.edu.sa
}

How to cite this paper: Al-Tuwairqi, S.M., Aloosh, B.K. and Bagies, R.A. (2019) The Future of Snapchat: A Mathematical Model. Journal of Applied Mathematics and Physics, 7, 841-860.

https://doi.org/10.4236/jamp.2019.74057

Received: March 8, 2019

Accepted: April 16, 2019

Published: April 19, 2019

Copyright $\odot 2019$ by author(s) and Scientific Research Publishing Inc. This work is licensed under the Creative Commons Attribution International License (CC BY 4.0).

http://creativecommons.org/licenses/by/4.0/

\begin{abstract}
Saudi Arabia has become one of the leading top five countries based on the number of Snapchat users as of October 2018. In this project, we build a novel mathematical model to explore the future of Snapchat in general and in Saudi Arabia particularly. The model incorporates the trend of "famous Snapchatters" that is highly observed in Saudi Arabia. The model is governed by a system of nonlinear differential equations. We analyze the system qualitatively and numerically. As a result, three equilibrium points are obtained. By considering their stability, we outline different possible scenarios for the future of Snapchat. Moreover, parameter analysis is performed to investigate key parameters in the model. Furthermore, an online survey is conducted to estimate the values for the parameters in the model to explore which scenario is likely to happen in Saudi Arabia.
\end{abstract}

\section{Keywords}

Mathematical Model, Stability, Snapchat, Saudi Arabia

\section{Introduction}

Snapchat is a multimedia and direct messaging application that enables users to send quick pictures, videos and messages to other users. These "Snaps" are only available to be viewed for a short time span before it is deleted permanently. This feature perhaps makes it, for some users, different and more attractable than other social network applications. Snaps posted by a user can be viewed either by "Friends" or "Followers". Friends add each other to their contact list in order to view each other snaps. However, followers may view the snaps of users they follow without being added to their contact list. It is not easy to find friends on Snapchat without knowing their username. However, Snapchat made it easy to add friends using their phone numbers under a service called "Find Friends". One final feature of Snapchat worth mentioning is "My Story" where snaps 
posted there last for twenty-four hours to be seen by friends and followers before it disappears.

Usage of Snapchat has been grown rapidly ever since its initiation in 2011 by its founders who were three undergraduates at Stanford University [1]. From 2012 to 2015, Snapchat has shown an approximated growth of 90 million users [2]. Also, a Business Insider article in 2017 has reported that Snapchat has collected about 158 million users that would use the app each day for an average of 25 - $30 \mathrm{~min}$ [3], which shows their satisfaction with the app. This frequent use of Snapchat has made it one of the top messaging apps like Facebook messaging and SMS [4].

The rise of Snapchat led researchers to investigate its impact on users: in particular, how individuals use and value Snapchat, what do they share, and with whom [5]. Also, another study was mainly concerned about how Snapchat behaviors influenced young adults interpersonal relationships [6]. Moreover, in a recent study, that was conducted on college students indicated there is a relationship between students needs and Snapchat addiction, intensity, and exhibitionism [7].

Saudi Arabia has become one of the leading top five countries based on the number of Snapchat users as of October 2018 [8]. In this region, some researchers were concerned in exploring how individuals' awareness toward privacy for online social networks (Snapchat) appose to their protective actions [9]. Moreover, in [10], the study was about examining how Saudi youth engagements with online social networks are formed and constrained within the cultural and religious aspects of this region. Meanwhile, others were assessing the effectiveness of Snapchat in raising the awareness of breast cancer among Saudi female students in the Dammam region [11].

To our knowledge, no one has searched the future of Snapchat in Saudi Arabia based on present data, especially, as a mathematical model. In this paper, we build a mathematical model to predict the future of Snapchat. This work is motivated by a similar study that was concerned about the future of Facebook [12]. The difference between our Snapchat model and the Facebook model is that we include a new class which represents the famous individuals within the population. These individuals are either famous and use Snapchat or became famous after being active users of Snapchat. In Saudi Arabia, the trend of "famous Snapchatters" is highly observable among its population. Therefore, our aim here is to investigate the role of the famous class in the growth of Snapchat generally and in Saudi Arabia particularly. Moreover, we aim to explore the future of Snapchat in Saudi Arabia based on data drawn from an online survey. This paper is organized as follows. In Section 2, we formulate a Snapchat mathematical model. We analyze the model qualitatively by obtaining the equilibrium points in Section 3 and examine its stability in Section 4. Moreover, three possible scenarios for the future of Snapchat are illustrated numerically in Section 5. Also, parameter analysis is demonstrated in Section 6 to see the influence of each parameter on the dynamics of Snapchat model. Furthermore, 
in Section 7 we present a Snapchat online survey conducted in Saudi Arabia and use its data to estimate the model's parameters in order to predict the future of Snapchat in Saudi Arabia. Finally, in Section 8 we give a brief conclusion.

\section{Mathematical Model}

The dynamics of Snapchat may be formulated as a mathematical model by first assuming that the population is divided into four distinct groups: Susceptible $(S)$, Infected $(I)$, Removed $(R)$ and Famous $(F)$. Susceptible refers to those who are not currently members of Snapchat, but there is a possibility that they may join at any time. Whereas, infected refers to those who are currently members of Snapchat and can recruit susceptibles to join as well. However, Removed refers to those who no longer use Snapchat. Finally, Famous refers to those who are famous and using Snapchat.

Snapchat can only thrive if it has active members, in this section, we will investigate the flow of individuals by analyzing the mathematical model to predict a possible future of Snapchat.

We assume that the typical flow of individuals from one group to another is as follows. Firstly, individuals who become older than 10 years are considered to be susceptibles and enter the model with constant enter rate. As for individuals who are older than 70 years are assumed to exit the model with constant exit rate, as well as those who leave the population as a result of death. For simplicity both enter and exit rates are assumed to be equal. Also, the exit rate will affect all groups equally. Secondly, we assume that susceptibles move to the infected group due to active users of Snapchat from family and friends, and also due to the need to follow some famous Snapchatters. In addition, active Snapchatters may gain more followers and become famous and move to the famous group. On the other hand, users of Snapchat may lose interest over time in Snapchat due to the influence of their family or friends who are no longer using Snapchat, and hence move to the removed group. However, the removed individuals may over time be subject to rejoin Snapchat again, and thus regain susceptibility; this may happen when some friends move away, so the only way to keep daily contact is through Snapchat. From the above assumptions, we may define the model's parameters and variables as follows :

$S(t)=$ Number of susceptibles at time $t$,

$I(t)=$ Number of infected at time $t$,

$R(t)=$ Number of removed at time $t$,

$F(t)=$ Number of famous at time $t$,

$b=$ Per-capita infection rate $\left[\right.$ time $^{-1} \cdot$ individuals $\left.^{-1}\right]$,

$a=$ Per-capita removed rate $\left[\right.$ time $^{-1} \cdot$ individuals $\left.^{-1}\right]$,

$c=$ Per-capita famous rate $\left[\right.$ time $^{-1} \cdot$ individuals $^{-1}$ ],

$d=$ Rate at which infected individuals become famous [ $\left[\right.$ ime $^{-1}$,

$v=$ Rate at which removed individuals regain susceptibility [time ${ }^{-1}$,

$\mu=$ Per-captia enter and exit rate $\left[\right.$ time $\left.^{-1}\right]$.

Note that all parametes are assumed to be positive, whereas the state variables 
are assumed to be nonnegative.

The dynamics of the model are illustrated in Figure 1, which are governed by the following system of nonlinear ordinary differential equations:

$$
\begin{gathered}
S^{\prime}=\mu+v R-b S I-c S F-\mu S \\
I^{\prime}=b S I+c S F-d I-\mu I-a I R \\
R^{\prime}=a I R-v R-\mu R \\
F^{\prime}=d I-\mu F
\end{gathered}
$$

Let $N(t)$ be the population size in this model, that is, $N(t)=S(t)+I(t)+R(t)+F(t)$. Also, let $N_{0}$ be the initial population size. By adding all the Equations in (1)-(4), we obtain the following initial-value problem:

$$
N^{\prime}=\mu(1-N), N(0)=N_{0}
$$

This can be solved for $N$ to obtain $N(t)=-\frac{\mathrm{e}^{-\mu t}}{\mu}\left(N_{0}-1\right)+1$. Thus, over a long period of time, the population approaches size 1 . Hence, we choose to study the system in the following region:

$$
\Gamma=\{(S, I, R, F): S+I+R+F \leq 1, S>0, I \geq 0, R \geq 0, F \geq 0\} .
$$

\section{Equilibrium Points}

To find the equilibrium points of the system, we equate the right-hand side of Equations (1)-(4) to zero, that is,

$$
\begin{gathered}
\mu+v R-b S I-c S F-\mu S=0 \\
b S I+c S F-d I-\mu I-a I R=0 \\
a I R-v R-\mu R=0 \\
d I-\mu F=0
\end{gathered}
$$

From Equation (7) we have, $R(a I-v-\mu)=0$, thus, either $R=0$ or $a I-v-\mu=0$. If $R=0$, then from (8), we get $F=\frac{d}{\mu} I$. By substituting for $R$ and $F$ in (6) we obtain $I\left(b S+c S \frac{d}{\mu}-d-\mu\right)=0$, thus, either $I=0$ or

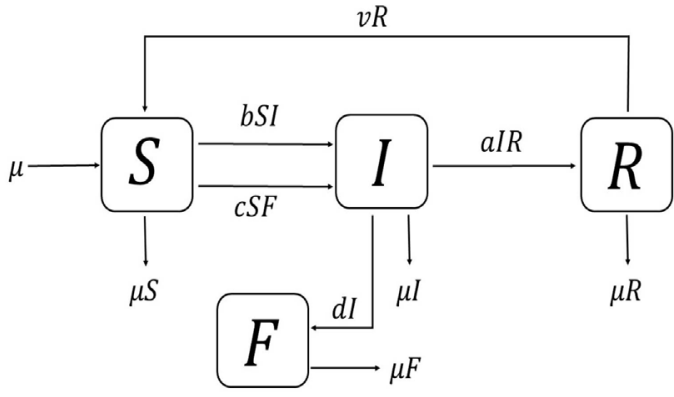

Figure 1. Flow chart of the Snapchat mathematical model. 
$b S+c S \frac{d}{\mu}-d-\mu=0$. If $I=0$ then $F=0$. Substituting in (5), we have $\mu-\mu S=0$, thus, $S=1$. Hence, the first equilibrium point is $E_{0}=(1,0,0,0)$.

Next, if $I \neq 0$, then $b S+c S \frac{d}{\mu}-d-\mu=0$, which yield $S=\frac{\mu(d+\mu)}{b \mu+d c}$. Moreover, from (5) we have $I=\frac{\mu^{2}(1-S)}{S(b \mu+c d)}$. By substituting for $I$ in (8) we obtain $F$ in terms of $I$. Hence, the second equilibrium point is $E_{1}=\left(S_{1}, I_{1}, 0, F_{1}\right)$, where

$$
S_{1}=\frac{\mu(\mu+d)}{\mu b+c d}, I_{1}=\frac{\mu^{2}\left(1-S_{1}\right)}{(b \mu+c d) S_{1}}, F_{1}=\frac{d I_{1}}{\mu} .
$$

Finally, if $R \neq 0$, then from (7) we get $I=\frac{v+\mu}{a}$, which implies, from (8), that $F=\frac{d(v+\mu)}{a \mu}$. By substituting for $I$ and $F$ in (6), we have $I\left(b S+c S \frac{d}{\mu}-d-\mu-a R\right)=0$, and since $I \neq 0$, then

$b S+c S \frac{d}{\mu}-d-\mu-a R=0$, which implies that $R=\frac{1}{a}\left(S\left(b+\frac{c d}{\mu}\right)-d-\mu\right)$. Now, when substituting for $I, F$ and $R$ in (5) we get $S$. Hence, the third equilibrium point is $E^{*}=\left(S^{*}, I^{*}, R^{*}, F^{*}\right)$, where

$$
\begin{gathered}
S^{*}=\frac{a \mu-v(\mu+d)}{c d+a \mu+b \mu}, I^{*}=\frac{v+\mu}{a}, F^{*}=\frac{d(v+\mu)}{a \mu}, \\
R^{*}=\frac{1}{a \mu(c d+a \mu+b \mu)}(b \mu+c d)(a \mu-v(d+\mu)-(a \mu+b \mu+c d)(\mu+d) \mu) .
\end{gathered}
$$

To summarize the above, we found three equilibrium points and they exist with the following conditions:

1) $E_{0}=\left(S_{0}, I_{0}, R_{0}, F_{0}\right)=(1,0,0,0)$, where $E_{0}$ exists always since all the values of $S_{0}, I_{0}, R_{0}$ and $F_{0}$ are nonnegative. We denote this equilibrium point by free-users since the infected class is zero.

2) $E_{1}=\left(S_{1}, I_{1}, 0, F_{1}\right)$, where $S_{1}=\frac{\mu(\mu+d)}{\mu b+c d}, \quad I_{1}=\frac{\mu^{2}\left(1-S_{1}\right)}{(b \mu+c d) S_{1}}, \quad F_{1}=\frac{d I_{1}}{\mu}$, which also exists without any condition since $S_{1}<1$. We refer to this equilibrium point as persistent-users since the removed class is zero.

$$
\begin{gathered}
\text { 3) } E^{*}=\left(S^{*}, I^{*}, R^{*}, F^{*}\right) \text {, where } S^{*}=\frac{a \mu-v(\mu+d)}{c d+a \mu+b \mu}, I^{*}=\frac{v+\mu}{a}, \\
R^{*}=\frac{1}{a \mu(c d+a \mu+b \mu)}[(b \mu+c d)(a \mu-v(d+\mu))-(a \mu+b \mu+c d)(\mu+d) \mu], \\
F^{*}=\frac{d(v+\mu)}{a \mu} .
\end{gathered}
$$


This point exists if $K_{2}=\frac{a \mu}{v(\mu+d)}>1$ and

$K_{3}=\frac{(b \mu+c d)(a \mu-v(d+\mu))}{\mu(\mu+d)(a \mu+b \mu+c d)}>1$. We name this equilibrium point as an endemic point since all classes exist together.

\section{Stability}

Here, we use the linearization method [13] to investigate the stability of the model. First, we will find the Jacobian matrix for the system (1)-(4) in order to obtain the eigenvalues for the three equilibrium points.

$$
J(S, I, R, F)=\left[\begin{array}{cccc}
-b I-c F-\mu & -b S & v & -c S \\
b I+c F & b S-d-\mu-a R & -a I & c S \\
0 & a R & a I-v-\mu & 0 \\
0 & d & 0 & -\mu
\end{array}\right]
$$

\section{1) Free-users equilibrium point $E_{0}$ :}

\section{Theorem 1}

The free-users equilibrium point $\left(E_{0}\right)$ is locally asymptotically stable if $K_{0}=\frac{b}{2 \mu+d}<1$ and $K_{1}=\frac{\mu b+c d}{\mu(\mu+d)}<1$.

Proof.

Substituting $E_{0}$ into $J(S, I, R, F)$ to obtain the following:

$$
J\left(E_{0}\right)=J(1,0,0,0)=\left[\begin{array}{cccc}
-\mu & -b & v & -c \\
0 & b-d-\mu & 0 & c \\
0 & 0 & -v-\mu & 0 \\
0 & d & 0 & -\mu
\end{array}\right] .
$$

The eigenvalues of this matrix are: $\lambda_{1}=-\mu, \lambda_{2}=-v-\mu$ and $\lambda_{3,4}$ satisfy the characteristic equation:

$$
a_{2} \lambda^{2}+a_{1} \lambda+a_{0}=0
$$

where $a_{2}=1, a_{1}=2 \mu+d-b$ and $a_{0}=\mu^{2}+\mu(d-b)-c d$. Now, for $E_{0}$ to be locally asymptotically stable, the eigenvalues must be negative. It is clear that $\lambda_{1}$ and $\lambda_{2}$ are negative. However, for $\lambda_{3}$ and $\lambda_{4}$, to be negative, we must show that $a_{1}, a_{2}$ and $a_{0}$ are all positive. Clearly, $a_{2}$ is positive, as for $a_{1}$ and $a_{0}$ they are positive if $K_{0}=\frac{b}{2 \mu+d}<1$ and $K_{1}=\frac{\mu b+c d}{\mu(\mu+d)}<1$ respectively.

\section{2) Persistent-users equilibrium point $E_{1}$ :}

\section{Theorem 2}

The persistent-user equilibrium point $\left(E_{1}\right)$ is locally asymptotically stable if $K_{1}=\frac{\mu b+c d}{\mu(\mu+d)}>1$ and

$$
K_{3}=\frac{(b \mu+c d)(a \mu-v(d+\mu))}{\mu(\mu+d)(a \mu+b \mu+c d)}<1
$$




\section{Proof.}

Evaluate the Jacobian at the equilibrium point $E_{1}$, we obtain

$$
J\left(S_{1}, I_{1}, 0, F_{1}\right)=\left[\begin{array}{cccc}
-b I_{1}-c F_{1}-\mu & -b S_{1} & v & -c S_{1} \\
b I_{1}+c F_{1} & b S_{1}-d-\mu & -a I & c S_{1} \\
0 & 0 & a I_{1}-v-\mu & 0 \\
0 & d & 0 & -\mu
\end{array}\right] .
$$

By solving the characteristic equation $|J-\lambda I|=0$, we obtain one eigenvalue explicitly, $\lambda_{1}=a I_{1}-v-\mu$, and the others satisfy the equation:

$$
a_{3} \lambda^{3}+a_{2} \lambda^{2}+a_{1} \lambda+a_{0}=0,
$$

where

$$
\begin{gathered}
a_{3}=1, \\
a_{2}=\frac{b \mu\left(b \mu+2 c d+\mu d+\mu^{2}\right)+c d\left(c d+d^{2}+3 \mu d+2 \mu^{2}\right)}{(d+\mu)(c d+b \mu)}, \\
a_{1}=\frac{b d \mu\left(b \mu+2 c d+4 c \mu-d \mu-2 \mu^{2}\right)+b \mu^{3}(2 b-\mu)+c^{2} d^{2}(d+2 \mu)}{(d+\mu)(c d+b \mu)}, \\
a_{0}=\mu c d+b \mu^{2}-d \mu^{2}-\mu^{3} .
\end{gathered}
$$

By substituting for $I_{1}$ in $\lambda_{1}$ we get $\lambda_{1}=\frac{a \mu\left(c d+b \mu-d \mu-\mu^{2}\right)}{(d+\mu)(c d+b \mu)}-v-\mu$, which is clearly negative if $K_{3}=\frac{(b \mu+c d)(a \mu-v(d+\mu))}{\mu(\mu+d)(a \mu+b \mu+c d)}<1$. To prove that $\lambda_{2}, \lambda_{3}$ and $\lambda_{4}$ are negative, we use Routh-Hurwitz Criterion [13]. We must satisfy the following conditions: $a_{i}>0, a_{2} a_{1}>a_{0}, i=0,1,2,3$. Clearly, $a_{3}, a_{2}$ are always positive, and $a_{0}>0$ is positive if $K_{1}=\frac{\mu b+c d}{\mu(\mu+d)}>1$. Now, evaluating

$$
\begin{aligned}
a_{2} a_{1}-a_{0}= & \left(b^{2} \mu^{2}+2 b c d \mu+c^{2} d^{2}+c d^{3}+2 c d^{2} \mu+c d \mu^{2}\right) \\
& \times\left(2 b^{2} \mu^{3}+4 b c d \mu^{2}+c^{2} d^{3}+2 c^{2} d^{2} \mu+c d^{2} \mu^{2}+c d \mu^{3}+b c d^{2} \mu\right. \\
& \left.+b^{2} d \mu^{2}+b c d^{2} \mu-\left(b d^{2} \mu^{2}+b d \mu^{3}\right)\right) /(d+\mu)^{2}(c d+b \mu)^{2} .
\end{aligned}
$$

Simplifying the under line terms, we obtain

$$
\begin{aligned}
& b^{2} d \mu^{2}+b c d^{2} \mu-\left(b d^{2} \mu^{2}+b d \mu^{3}\right) \\
& =\left(b d^{2} \mu^{2}+b d \mu^{3}\right)\left(\frac{b^{2} d \mu^{2}+b c d^{2} \mu}{b d^{2} \mu^{2}+b d \mu^{3}}-1\right) \\
& =\left(b d^{2} \mu^{2}+b d \mu^{3}\right)\left(\frac{b \mu+c d}{d \mu+\mu^{2}}-1\right) \\
& =\left(b d^{2} \mu^{2}+b d \mu^{3}\right)\left(K_{1}-1\right) .
\end{aligned}
$$

If $K_{1}>1$ then $a_{2} a_{1}>a_{0}$. Hence, $\lambda_{2}, \lambda_{3}$ and $\lambda_{4}$ are negative by 
Routh-Hurwitz Criterion [13]. Thus, $E_{1}$ is locally asymptotically stable if: $K_{1}>1$ and $K_{3}<1$.

\section{3) Endemic equilibrium point $E^{*}$ :}

\section{Theorem 3}

The endemic equilibrium point $E^{*}$ is locally asymptotically stable provided $A_{i}>0$, where $i=0,1,2$ and $A_{1} A_{2}>A_{0}$. Here $A_{0}, A_{1}$ and $A_{2}$ are provided in the proof.

Proof.

Evaluate the Jacobian at the equilibrium point $E^{*}$, we obtain

$$
J\left(S^{*}, I^{*}, R^{*}, F^{*}\right)=\left[\begin{array}{cccc}
-b I^{*}-c F^{*}-\mu & -b S^{*} & v & -c S^{*} \\
b I^{*}+c F^{*} & b S^{*}-d-\mu-a R^{*} & -a I^{*} & c S^{*} \\
0 & a R^{*} & 0 & 0 \\
0 & d & 0 & -\mu
\end{array}\right] .
$$

Here we have used that $a I^{*}-v-\mu=0$ since $R^{*} \neq 0$. By solving the characteristic equation $|J-\lambda I|=0$, we obtain one eigenvalue explicitly, $\lambda_{1}=-\mu$, and the others satisfy the equation:

$$
\lambda^{3}+A_{2} \lambda^{2}+A_{1} \lambda+A_{0}=0,
$$

where

$$
\begin{gathered}
A_{2}=b I^{*}+c F^{*}+a R^{*}+2 \mu+d-b S^{*}, \\
A_{1}=a^{2} I^{*} R^{*}+\left(b I^{*}+c F^{*}+\mu\right)\left(d+\mu+a R^{*}\right)-S^{*}(c d+b \mu), \\
A_{0}=a^{2} \mu I^{*} R^{*}+a R^{*}\left(b I^{*}+c F^{*}\right)\left(a I^{*}-v\right) .
\end{gathered}
$$

It is easy to observe that $A_{0}>0$ since $a I^{*}-v=\mu$. Hence, by Routh-Hurwitz criterion, the local asymptotic stability of $E^{*}$ is guaranteed under the conditions stated in the theorem.

\section{Remark 1}

Note that if $E_{0}$ is stable, then $E_{1}$ is not stable and vice versa, since they have opposite conditions for stability. Also, one of the stability conditions of $E_{1}$ is the exact opposite to one of the existence conditions of $E^{*}$, which means that if $E^{*}$ exists then $E_{1}$ is unstable or if $E_{1}$ is stable then $E^{*}$ does not exist.

\section{Numerical Simulations}

In this section, we will demonstrate the numerical simulations of the model by solving the system numerically using Matlab. In addition, we will show the agreement of the qualitative results with the numerical simulations.

Firstly, we choose parameters to satisfy the conditions of the free-users equilibrium point $E_{0}$, namely, $K_{0}<1$ and $K_{1}<1$. We let $\mu=0.015, b=0.01, c=0.01, d=0.01, a=0.01$ and $v=0.01$. In Figure 2, we see that for different sets of initial conditions, the size of susceptibles eventually reaches the value of one, whereas, the size of the other compartments reaches zero. Thus, the behavior of the model in the long term reaches the equilibrium $E_{0}$. 


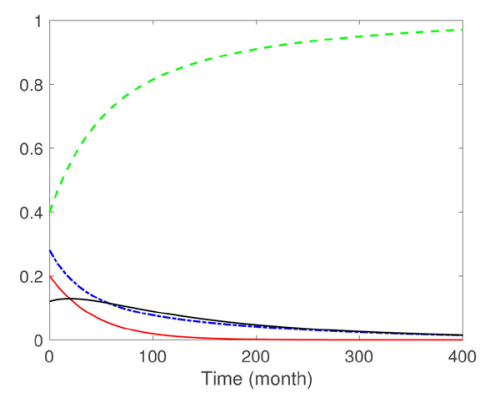

(a)

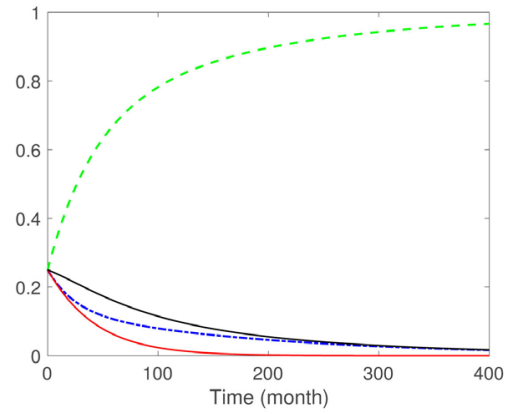

(b)

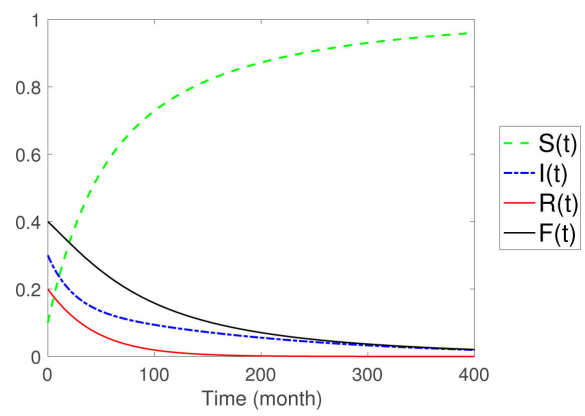

(c)

Figure 2. The dynamical behavior of the model with parameters satisfying conditions of $E_{0}$ with the following sets of initial conditions: (a) $(0.4,0.28,0.2,0.12)$, (b) $(0.25,0.25$, $0.25,0.25)$, (c) $(0.1,0.3,0.2,0.4)$.

Secondly, we choose $\mu=0.015, b=0.05, c=0.01, d=0.01, a=0.05 \quad$ and $v=0.01$ to satisfy the conditions of the persistent-users equilibrium point $E_{1}$, that is, $K_{1}>1$ and $K_{3}<1$. Figure 3 illustrates the time variation of each compartment of the model with this set of parameters. The size of the removed compartment decreases with time until it reaches zero. However, the size of the other compartments fluctuates with time until it reaches an equilibrium value. Hence, for different initial conditions, we see that the model eventually approaches the equilibrium $E_{1}=(0.4412,0.3353,0,0.2235)$.

Finally, we change the parameters to the following values: $\mu=0.015, b=0.05$, $c=0.05, d=0.01, a=0.07$ and $v=0.01$ which satisfy $K_{2}>1$ and $K_{3}>1$, the conditions of the endemic equilibrium point $E^{*}$. Figure 4 shows the existence of all compartments of the model with variations in their sizes. The removed compartment eventually has the least size, while the susceptible compartment holds the largest size. Thus, the long-term behavior of the model reaches the equilibrium $E^{*}=(0.3478,0.3571,0.0569,0.2381)$ for different initial conditions.

The numerical simulations presented in this section, demonstrate good agreement with the qualitative results given in Section 4.

\section{Parameter Analysis}

To have a better understanding of the impact of the parameters on the dynamics of the model, we vary the parameters in the simulations. In this section, we 


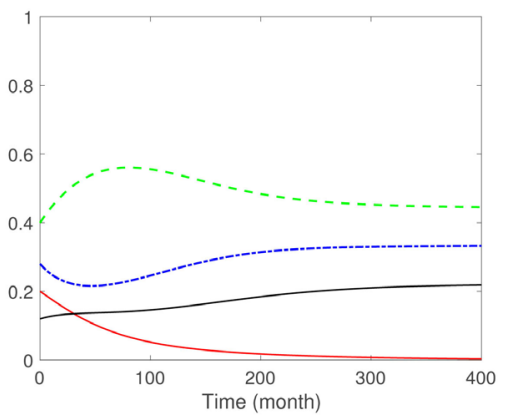

(a)

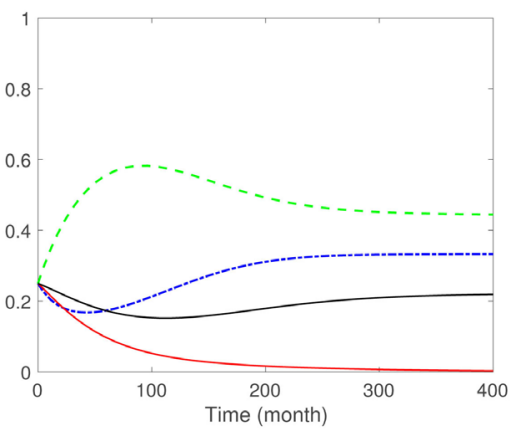

(b)

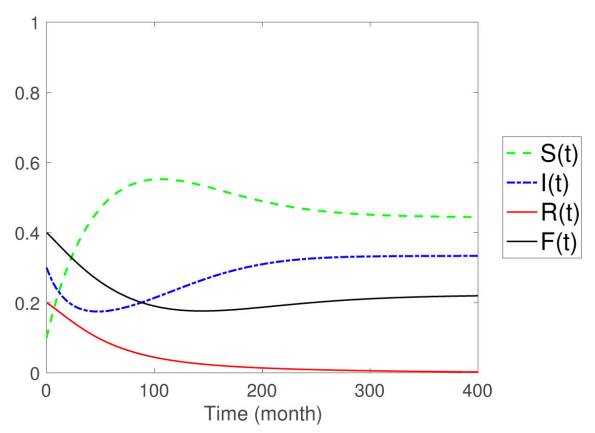

(c)

Figure 3. The dynamical behavior of the model with parameters satisfying conditions of $E_{1}$ with the following sets of initial conditions: (a) $(0.4,0.28,0.2,0.12)$; (b) $(0.25,0.25$, $0.25,0.25)$; (c) $(0.1,0.3,0.2,0.4)$.

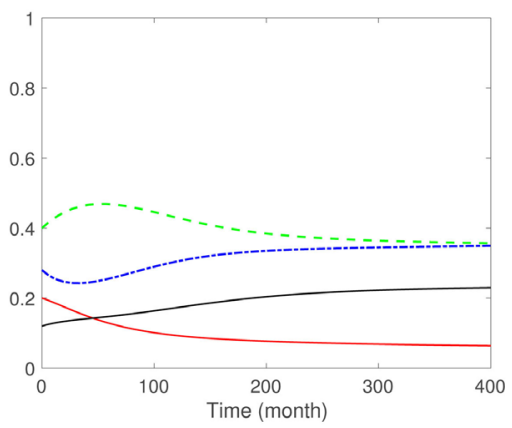

(a)

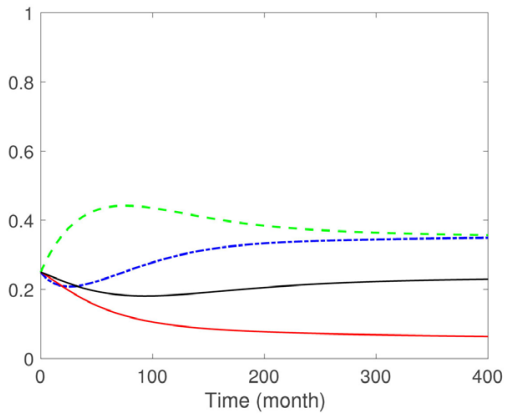

(b)

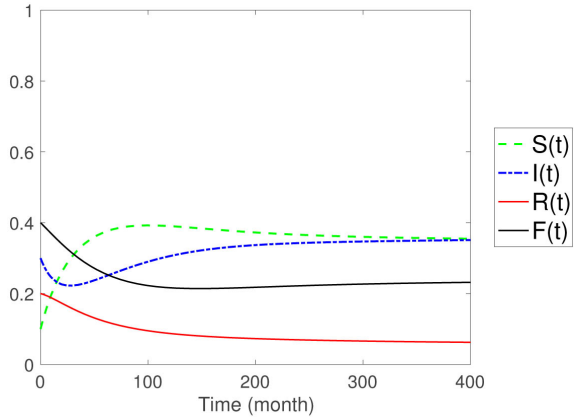

(c)

Figure 4. The dynamical behavior of the model with parameters satisfying conditions of $E^{*}$ with the following sets of initial conditions: (a) $(0.4,0.28,0.2,0.12)$; (b) $(0.25,0.25$, $0.25,0.25)$; (c) $(0.1,0.3,0.2,0.4)$. 
analyze the system's parameters by choosing one parameter to vary while keeping the other parameters fixed. Our goal is mainly to see the effect of the varying parameter on the infected compartment in relation to another compartment by using numerical simulations. Throughout the simulations, the initial conditions are set as follows:

$$
S(0)=0.4, I(0)=0.28, R(0)=0.2, F(0)=0.12
$$

First, we vary the parameter $b$, the per-capita infection rate, and fix all other parameters to be: $\mu=0.01, c=0.01, d=0.01, a=0.01, v=0.01$. Figure 5(a) shows the relation between the infected and susceptible compartments while varying $b$. If $b$ is small, the size of the infected class decreases while the susceptible increase with time. However, when the rate $b$ increases, the size of the infected class starts to increase, whereas the susceptibles decrease until they both reach an equilibrium value. This indicates that there is a critical value of $b$ where the infected class starts to rise. Similar results are given in Figure 5(b) for varying the parameter $c$, the per-capita famous rate and fixing the other parameters to be: $\mu=0.01, b=0.01, d=0.01, a=0.01, v=0.01$. As for the parameter $a$, the per-capita removed rate, we plot the infected class against the removed class. We fix all parameters to be: $\mu=0.01, b=0.05, c=0.05, d=0.01$, $v=0.01$. Figure 6 illustrates that for small values of $a$, the infected class increases while the removed class decreases with time. However, as a increases, the removed class starts to increase, and the infected class rapidly decreases until they reach a certain value. Hence, the parameter a has a critical value such that below this value the infected class increases.

Finally, we analyze the rate at which infected individuals become famous and the rate at which removed individuals regain susceptibility, that is, the parameters $d$ and $V$ respectively. We plot the relation between infected and famous compartments, which is illustrated in Figure 7 for fixed parameters: $\mu=0.01, b=0.05, c=0.05, d=0.01, a=0.01, v=0.01$. If the parameter $d$ is small, both compartments increase with time. As the parameter $d$ increases, the infected class decreases rapidly, whereas, the famous class increases with time until they both reach an equilibrium value. Here, whether the parameter $d$ is

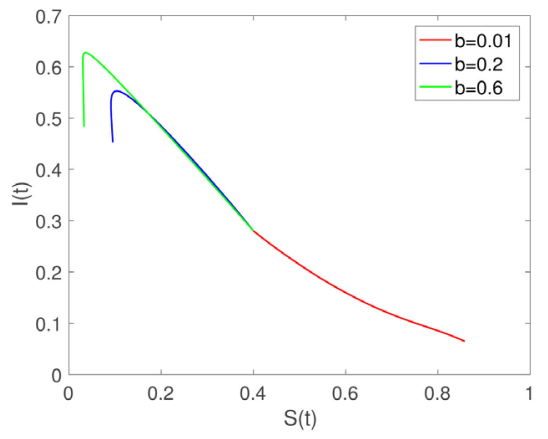

(a)

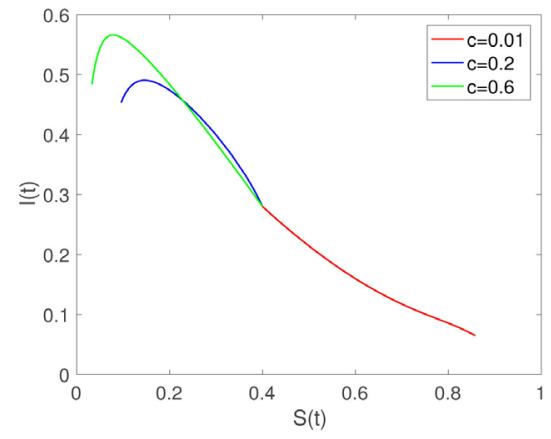

(b)

Figure 5. Phase plot of susceptible and infected compartments with varying (a) the parameter $b$ and (b) the parameter $c$, and fixing all other parameters. 


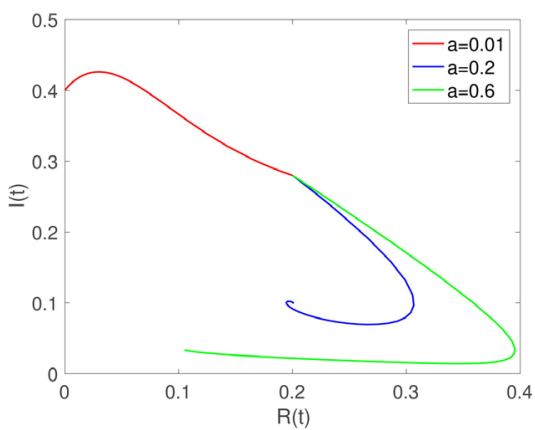

Figure 6. Phase plot of removed and infected compartments with varying the parameter a and fixing all other parameters.

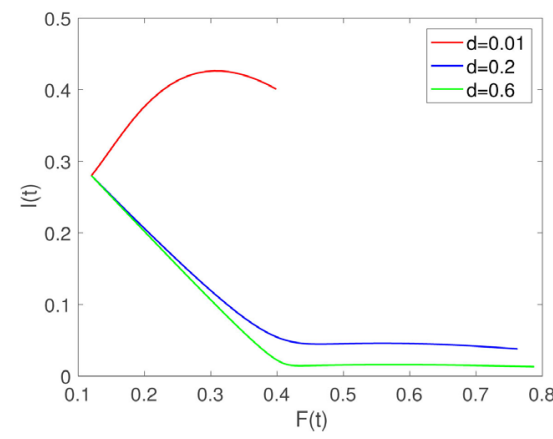

(a)

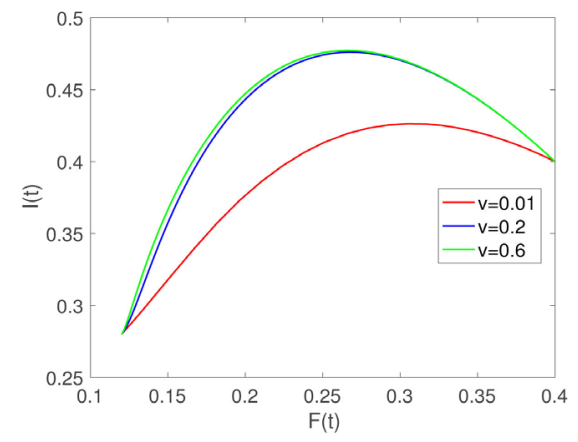

(b)

Figure 7. Phase plot of famous and infected compartments with varying (a) the parameter $d$ and (b) the parameter $v$, and fixing all other parameters.

small or large it reflects growth in Snapchat users since the increase in famous class means that Snapchatters are rising and becoming famous. On the other hand, As the parameter $v$ increases, both infected and famous classes increase until they reach a peak, then infected class starts to decay, whereas, famous class continues to increase with time (see Figure $7(b)$ ). Also, no matter what the value of the parameter $v$ is, an increase in Snapchat users occur whether they are ordinary or famous users. Indeed, since parameter $v$ indicates that removed individuals regain susceptibility again.

\section{The Future of Snapchat in Saudi Arabia}

In the previous sections, we illustrated three possible scenarios for the Snapchat mathematical model over a long period of time. The first scenario is that Snapchat becomes completely neglected by individuals in the population. On the other hand, the second scenario shows the potential persistence of Snapchat with a consistent amount of individuals being only users or susceptibles. Also, in the third scenario Snapchat continues to thrive in the population, but with some amount of individuals who no longer use the app.

In this section, we use the same mathematical model to predict a possible future of Snapchat in Saudi Arabia. This is accomplished by estimating the parameters in the model from statistical data gathered from an online survey 
conducted throughout the country of Saudi Arabia. We will explain further in the following subsections.

\subsection{Statistical Survey}

A cross-sectional online survey was carried out in Saudi Arabia (SA) on the $24^{\text {th }}$ of March till the $1^{\text {st }}$ of July 2018 . Our objective was to explore to what extent does active and nonactive users of Snapchat influence individuals towards using or removing the app. Also, to investigate the impact of famous Snapchatters on the use of Snapchat.

The survey was conducted through electronic social networks. Participants were asked to complete an online questionnaire. The number of participants who completed the questionnaire was $\mathrm{n}=1700$. The questionnaire was divided into two sections. The first section contained demographic questions about the participant's age, gender, living area and career. The second section included the participant's behavior towards using or non-using Snapchat. We have analyzed the data obtained from the study by using Excel (descriptive analysis). The results are demonstrated in this subsection.

The age distribution of the participants were 149 (9\%) adolescents, 971 (57\%) young adults, $512(30 \%)$ middle-aged adults, and 68 (4\%) older adults. Thus, the majority of the participants were young adults. Also, most of them were female, 1524 (90\%). Moreover, they were classified according to their career as follows: 584 individuals (34\%) were students, 554 individuals (33\%) were employees, 102 individuals (6\%) were unemployed, 336 individuals (20\%) were housewives, and 124 individuals $(7 \%)$ were retired. In terms of participant's living area, the majority (83\%) lived in west of SA, followed by (9\%) participants lived in the center of SA, (3\%) lived in the south of SA, (3\%) lived in the east of SA, and (2\%) lived in the north of SA (see Table 1).

The survey shows that (80\%) of the participants use Snapchat. Table 2 reveals the investigations of behavior among participants who presently use Snapchat. It is found that 1283 (94\%) participants knew about Snapchat from family and friends. Also, 162 (12\%) participants reported that their reason for using Snapchat was to follow a famous Snapchatter. However, $56 \%$ of the participants follow an average of 10 famous Snapchatters, whom 7 out of the 10, they think that Snapchat was the reason behind their fame. As for which snaps they see more, $850(62.5 \%)$ participants stated that they see snaps from family and friends more compared to $85(6 \%)$ participants who see snaps posted by famous Snapchatters more, and the rest of the participants, 427 (31.5\%), see snaps from both family and famous Snapchatters equally. Regarding the influence of famous Snapchatters, $775(57 \%)$ of participants said that their snaps provide useful information for them, and 672 (49\%) stated that following famous Snapchatters keep them updated with what is new in different areas. On the other hand, the study shows that only $25 \%$ prefer a particular consumer product, and $19 \%$ prefer a specific brand as a result of the recommendation and acquaintance of famous Snapchatters. Finally, 858 (63\%) participants consider Snapchat as a useful tool 
Table 1. Demographic data of participants in Snapchat survey.

\begin{tabular}{|c|c|c|c|c|}
\hline & & Frequency & Percent & Cumulative percent \\
\hline \multirow[t]{8}{*}{ Age } & $10-12$ years & 5 & 0.3 & 0.3 \\
\hline & 13 - 15 years & 23 & 1.4 & 1.7 \\
\hline & 16 - 18 years & 121 & 7.1 & 8.8 \\
\hline & 19 - 29 years & 632 & 37.2 & 46 \\
\hline & 30 - 39 years & 339 & 19.9 & 65.9 \\
\hline & $40-49$ years & 309 & 18.2 & 84.1 \\
\hline & $50-59$ years & 203 & 11.9 & 96 \\
\hline & $>60$ years & 68 & 4 & 100 \\
\hline \multirow[t]{2}{*}{ Gender } & Male & 175 & 10.3 & 10.3 \\
\hline & Female & 1525 & 89.7 & 100 \\
\hline \multirow[t]{5}{*}{ Living Area } & North of Saudi Arabia & 25 & 1.5 & 1.5 \\
\hline & South of Saudi Arabia & 58 & 3.4 & 4.9 \\
\hline & East of Saudi Arabia & 56 & 3.3 & 8.2 \\
\hline & West of Saudi Arabia & 1407 & 82.8 & 91 \\
\hline & Center of Saudi Arabia & 154 & 9.1 & 100 \\
\hline \multirow[t]{7}{*}{ Career } & Student & 584 & 34.4 & 34.4 \\
\hline & Employee in privet sector & 365 & 21.5 & 55.9 \\
\hline & Employee in government sector & 147 & 8.6 & 64.5 \\
\hline & Owned business & 42 & 2.5 & 67 \\
\hline & House wife & 336 & 19.8 & 86.8 \\
\hline & Looking for a job & 102 & 6 & 92.8 \\
\hline & Retired & 124 & 7.3 & 100 \\
\hline
\end{tabular}

to gain helpful information, in particular, 593 (44\%) use Snapchat as an educational tool. However, 515 (38\%) stated that Snapchat consumes a lot of their time.

As for participants who are not currently using Snapchat, $8 \%$ of them reported that they do not know the app, 58\% said that they knew the app but have not used it, and $34 \%$ stated that they used the app but chose to remove it. Moreover, from among those who removed the app, 5\% declared the reason was that their family and friends no longer use the app. On the other hand, $18 \%$ said that they would reinstall Snapchat again to follow some famous Snapchatter. Finally, when participants were asked if they will rethink of using Snapchat, 11\% said yes, $32 \%$ said no and $57 \%$ stated that they do not know (see Table 3 ). 
Table 2. Characteristics and behavior among participants who are users of Snapchat.

\begin{tabular}{|c|c|c|c|c|}
\hline & & Frequency & Percent & $\begin{array}{l}\text { Cumulative } \\
\text { percent }\end{array}$ \\
\hline \multirow[t]{2}{*}{ Doyou use Snapchat now? } & Yes & 1362 & 80.1 & 80.1 \\
\hline & No & 338 & 19.9 & 100 \\
\hline \multirow[t]{2}{*}{$\begin{array}{l}\text { Did you know about Snapchat from a family } \\
\text { member, friend, colleague or other people? }\end{array}$} & Yes & 1283 & 94.2 & 94.2 \\
\hline & No & 79 & 5.8 & 100 \\
\hline \multirow[t]{2}{*}{$\begin{array}{l}\text { Was following a famousSnapchatter the } \\
\text { reason behind using Snapchat? }\end{array}$} & Yes & 162 & 11.9 & 11.9 \\
\hline & No & 1200 & 88.1 & 100 \\
\hline \multirow[t]{2}{*}{$\begin{array}{l}\text { How many famous Snapchattersyou often } \\
\text { follow on Snapchat? }\end{array}$} & $\begin{array}{l}\text { Between } \\
1 \text { and } 10\end{array}$ & 757 & 56 & 56 \\
\hline & 0 & 605 & 44 & 100 \\
\hline \multirow[t]{2}{*}{$\begin{array}{l}\text { How many famous Snapchatters from whom } \\
\text { you follow, do you think that Snapchat } \\
\text { was the reason behind their fame? }\end{array}$} & $\begin{array}{l}\text { Between } \\
1 \text { and } 7\end{array}$ & 757 & 56 & 56 \\
\hline & 0 & 605 & 44 & 100 \\
\hline \multirow[t]{3}{*}{ Whom do you see their snaps more? } & $\begin{array}{l}\text { Family and } \\
\text { friends }\end{array}$ & 850 & 62.4 & 62.4 \\
\hline & $\begin{array}{c}\text { Famous } \\
\text { Snapchatters }\end{array}$ & 85 & 6.2 & 68.6 \\
\hline & Both & 427 & 31.4 & 100 \\
\hline \multirow[t]{2}{*}{ Do you use Snapchat as an educational tool? } & Yes & 593 & 43.5 & 43.5 \\
\hline & No & 769 & 56.5 & 100 \\
\hline \multirow[t]{2}{*}{ Does Snapchat consume a lot of your time? } & Yes & 515 & 37.8 & 37.8 \\
\hline & No & 847 & 62.2 & 100 \\
\hline \multirow[t]{2}{*}{$\begin{array}{l}\text { Do you consider Snapchat a useful tool to } \\
\text { gain helpful information needed for your life? }\end{array}$} & Yes & 858 & 63 & 63 \\
\hline & No & 504 & 37 & 100 \\
\hline \multirow[t]{2}{*}{$\begin{array}{l}\text { Do you think famous Snapchatters whom } \\
\text { you follow provide useful information for you? }\end{array}$} & Yes & 775 & 56.9 & 56.9 \\
\hline & No & 587 & 43.1 & 100 \\
\hline \multirow[t]{2}{*}{$\begin{array}{l}\text { Do you follow famous Snapchatters to keep } \\
\text { updated with what is new in different areas? }\end{array}$} & Yes & 672 & 49.3 & 49.3 \\
\hline & No & 690 & 50.7 & 100 \\
\hline \multirow{2}{*}{$\begin{array}{l}\text { Is your preference for a consumer product } \\
\text { over another because of the recommendation } \\
\text { of a famous Snapchatter? }\end{array}$} & Yes & 345 & 25.3 & 25.3 \\
\hline & No & 1017 & 74.7 & 100 \\
\hline \multirow{2}{*}{$\begin{array}{l}\text { Do you find yourself preferring a brand } \\
\text { over another because a famous Snapchatter } \\
\text { is more acquaintance with this brand? }\end{array}$} & Yes & 262 & 19.2 & 19.2 \\
\hline & No & 1100 & 80.8 & 100 \\
\hline
\end{tabular}


Table 3. Characteristics and behavior among participants who are not users of Snapchat.

\begin{tabular}{|c|c|c|c|c|}
\hline & & Frequency & Percent & $\begin{array}{c}\text { Cumulative } \\
\text { percent }\end{array}$ \\
\hline \multirow[t]{3}{*}{ The reason for not using Snapchat is. } & $\begin{array}{c}\text { You do not } \\
\text { know Snapchat }\end{array}$ & 26 & 7.7 & 7.7 \\
\hline & $\begin{array}{l}\text { You know } \\
\text { Snapchat, but you } \\
\text { have not used it }\end{array}$ & 197 & 58.3 & 66 \\
\hline & $\begin{array}{l}\text { You have used it, } \\
\text { but now youdo not }\end{array}$ & 115 & 34 & 100 \\
\hline \multirow{2}{*}{$\begin{array}{l}\text { Have you ever deleted Snapchat then } \\
\text { reinstalled it again in order to follow } \\
\text { some famous Snapchatters? }\end{array}$} & Yes & 20 & 17.4 & 17.4 \\
\hline & No & 95 & 82.6 & 100 \\
\hline \multirow{2}{*}{$\begin{array}{l}\text { Haveyou stopped using Snapchat } \\
\text { because no one in your family or } \\
\text { friends uses it anymore? }\end{array}$} & Yes & 6 & 5.2 & 5.2 \\
\hline & No & 109 & 94.8 & 100 \\
\hline \multirow{3}{*}{$\begin{array}{l}\text { If you were a previous Snapchat user, } \\
\text { but currently not, will you rethink of } \\
\text { using it again? }\end{array}$} & Yes & 13 & 11.3 & 11.3 \\
\hline & No & 37 & 32.2 & 43.5 \\
\hline & Do not know & 65 & 56.5 & 100 \\
\hline
\end{tabular}

\subsection{Future Predictions}

In this subsection, we will use the mathematical model formulated in Section 2 to predict a possible future of Snapchat in Saudi Arabia. The parameters in the model will be estimated from the data given in the previous survey. Here, we will distinguish between the entry and the exit rates. As mentioned before, Individuals who are ten years and older enter the model with a constant rate, $B$, however, individuals who are older than 70 years and who leave the population due to natural death exit the model with a constant rate, $\mu$. The estimation of the entry and exit rates are $B=0.066$ and $\mu=0.018$ per month respectively. These values are calculated according to the data presented in the Annual Yearbook 2017 published online by the General Authority of Statistics in Saudi Arabia [14]. As for the other parameters, we evaluate the infection rate, $b$, the famous rate, $c$, and the rate at which infected individuals become famous, $d$, from the percent of individuals who answered yes to some related questions in Table 2. Also, in the same manner, from Table 3 we compute the removed rate, $a$, and the rate at which removed individuals regain susceptibility, $v$. The values of the parameters are presented in Table 4.

Next, the proposed model in Section 2, with the parameters estimated here, is solved numerically to predict the long-term behavior of the solutions. Note that the entry rate is different from the exit rate. Therefore, Equation (1) changes to the form: 
Table 4. Estimation of model's parameters from Snapchat survey.

\begin{tabular}{lccc}
\hline \multicolumn{1}{c}{ Parameter question } & $\begin{array}{c}\text { Estimated } \\
\text { parameter }\end{array}$ & $\begin{array}{c}\text { Value } \\
\text { per year }\end{array}$ & $\begin{array}{c}\text { Value } \\
\text { per month }\end{array}$ \\
\hline $\begin{array}{l}\text { Did you know about Snapchat from a family } \\
\text { member, friend, colleague or other people? }\end{array}$ & $\hat{b}$ & 0.942 & 0.078 \\
$\begin{array}{l}\text { Was following a famousSnapchatter the reason } \\
\text { behind using Snapchat? }\end{array}$ & $\hat{c}$ & 0.12 & 0.01 \\
$\begin{array}{l}\text { How many famous Snapchattersyou often } \\
\text { follow on Snapchat? }\end{array}$ & $\hat{c}$ & 0.7 & 0.058 \\
$\begin{array}{l}\text { How many famous Snapchatters from whom you } \\
\text { follow, do you think that Snapchat was the } \\
\text { reason behind their fame? }\end{array}$ & & & \\
$\begin{array}{l}\text { Haveyou stopped using Snapchat because no } \\
\text { one in your family or friends uses it anymore? }\end{array}$ & $\hat{a}$ & 0.052 & 0.004 \\
$\begin{array}{l}\text { If you were a previous Snapchat user, } \\
\text { but currently not, will you rethink of using it again? }\end{array}$ & $\hat{v}$ & 0.565 & 0.047 \\
\hline
\end{tabular}

$$
S^{\prime}=B+v R-b S I-c S F-\mu S
$$

Figure 8 shows that the future of Snapchat in Saudi Arabia behaves as the persistent-users equilibrium point $E_{1}$. We see that the removed individuals will demolish as time increases. However, the size of famous class rises rapidly to an equilibrium level, being the largest size of all the compartments. Also, the size of active users compartment rises to an equilibrium level that is far low from the famous class. As for susceptibles, we see a rise at the beginning then a fall in their size until they reach an equilibrium level. These observations are apparent for different initial conditions.

From this exploratory simulations, we may conclude that Snapchat continues to thrive in Saudi Arabia. Also, Snapchat is considered as a useful tool for individuals to become famous within the population, which again contributes in the prosper of Snapchat. This trend of Snapchat is being exhibited nowadays within the population of Saudi Arabia.

\section{Conclusions}

In this work, we proposed a mathematical model to predict the future of Snapchat. The motivation of this work came from a similar study that was conducted for Facebook [12]. The difference between our Snapchat model and the Facebook model is that we included a new class which represents the famous individuals within the population. These individuals are either famous and use Snapchat or became famous after being active users of Snapchat. We aimed to investigate the role of famous class in the growth of Snapchat generally and particularly in Saudi Arabia since the trend of "famous Snapchatters" is noticeable among its population.

The mathematical model was analyzed qualitatively and numerically. First, we examined the model by assuming that the enter and exit rates are equal. We 


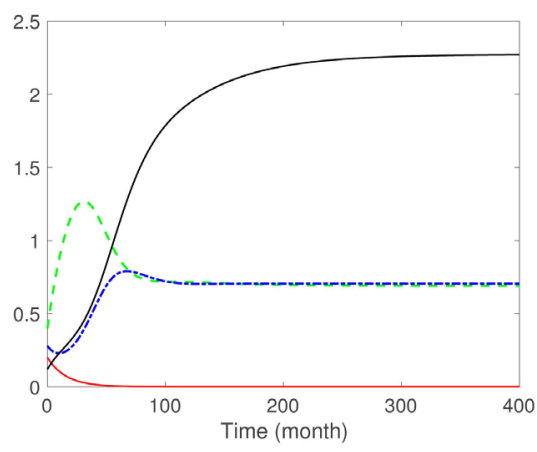

(a)

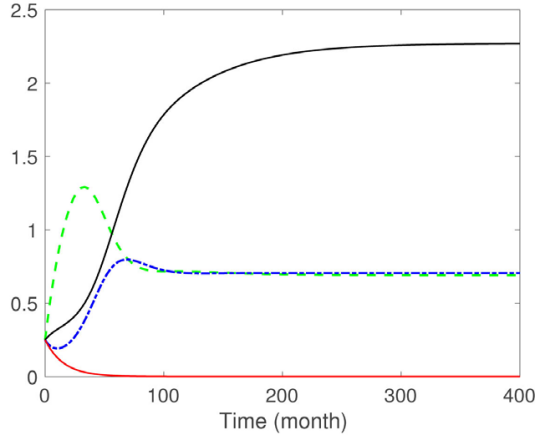

(b)

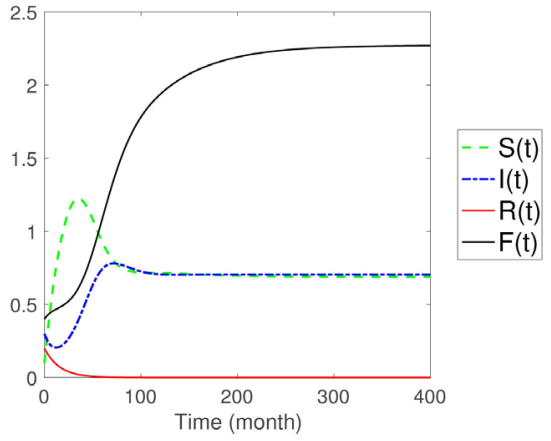

(c)

Figure 8. Time variation of the model compartments with parameters: $B=0.066, \mu=0.018, \hat{b}=0.078, \hat{c}=0.01, \hat{d}=0.058, \hat{a}=0.004$ and $\hat{v}=0.047$. and the following sets of initial conditions: (a) $(0.4,0.28,0.2,0.12)$, (b) $(0.25,0.25,0.25,0.25)$, (c) $(0.1,0.3,0.2,0.4)$.

obtained three equilibrium points for the model and proved their stability according to conditions satisfied by the parameters. Some examples of how this model behaves are given in Figures 2-4. It is possible that Snapchat may decline and disappear (Figure 2), or may continue to thrive as a result of either the drop in the size of removed individuals to zero (Figure 3) or the existence of individuals in all compartment at any given time (Figure 4). Each of these scenarios is predictable by letting the parameters of the model satisfy the existence and stability conditions of each case.

When analyzing the parameters in the model, we found that Snapchat blooms if the rates of active users and famous Snapchatters, who recruit individuals, reach above a specific amount (Figure 5). On the other hand, if the rate of non-users of Snapchat increases, then, with time, the size of Snapchat users falls, which leads to the decay of Snapchat (Figure 6). As for the rates at which individuals become famous or become susceptible again, we found that both rates lead to the growth of Snapchat. In particular, these rates contribute in either an increase in the size of active users or the size of famous Snapchatters or an increase in both (Figure 7).

One of the aims of this work was to predict the future of Snapchat in Saudi Arabia. Therefore, an online survey was conducted to help in the estimations of 
the parameters in the model. The survey showed that Snapchat is growing in Saudi Arabia since $80 \%$ of the participants in the study were using Snapchat. Moreover, the survey reflected the significant role of active users in recruiting new individuals, since $94 \%$ of the participant said that they knew about Snapchat from family and friends. Based on the estimated values of the parameters from the survey (Table 4) and the proposed mathematical model, exploratory simulations were generated numerically. It was observed that the future of Snapchat in Saudi Arabia might behave as the persistent-users equilibrium point $E_{1}$ from the general model in Section 2, but with a significant increase in the class of famous Snapchatters.

In conclusion, this exploratory study helped in gaining a better insight as for how Snapchat can continue to thrive and when will it disappears. Also, how Snapchat in Saudi Arabia gave rise to famous Snapchatters, who in return contributes to the prosper of Snapchat.

\section{Conflicts of Interest}

The authors declare no conflicts of interest regarding the publication of this paper.

\section{References}

[1] Lemay, D.J., Doleck, T. and Bazelais, P. (2017) Passion and Concern for Privacy as Factors Affecting Snapchat Use: A Situated Perspective on Technology Acceptance. Computers in Human Behavior, 75, 264-271. https://doi.org/10.1016/j.chb.2017.05.022

[2] Macmillan, D. and Rusli, E. (2014) Snapchat Is Said to Have More than 100 Million Monthly Active Users. The Wall Street Journal.

https://blogs.wsj.com/digits/2014/08/26/snapchat-said-to-have-more-than-100-milli on-monthly-active-users/

[3] Carson, B. (2017) Here's Everything You Need to Know about How Many People Are Using Snapchat. Business Insider.

[4] (2017) Most Popular Mobile Messaging Apps Worldwide as of January 2017, Based on Number of Active Users (in Millions).

https://www.statista.com/statistics/258749/most-popular-global-mobile-messengerapps/

[5] Piwek, L. and Joinson, A. (2016) “What Do They Snapchat About?” Patterns of Use in Time-Limited Instant Messaging Service. Computers in Human Behavior, 54, 358-367.

[6] Vaterlaus, J.M., Barnett, K., Roche, C. and Young, J.A. (2016) Snapchat Is More Personal: An Exploratory Study on Snapchat Behaviors and Young Adult Interpersonal Relationships. Computers in Human Behavior, 62, 594-601. https://doi.org/10.1016/j.chb.2016.04.029

[7] Punyanunt-Carter, N.M., Cruz, J.D.L. and Wrench, J.S. (2017) Investigating the Relationships among College Students' Satisfaction, Addiction, Needs, Communication Apprehension, Motives, and Uses and Gratifications with Snapchat. Computers in Human Behavior, 75, 870-875. https://doi.org/10.1016/j.chb.2017.06.034

[8] (2018) Leading Countries Based on Number of Snapchat Users as of October 2018 
(in Millions).

https://www.statista.com/statistics/315405/Snapchat-user-region-distribution/

[9] AlSagri, H.S. and AlAboodi, S.S. (2015) Privacy Awareness of Online Social Networking in Saudi Arabia. International Conference on Cyber Situational Awareness, Data Analytics and Assessment (CyberSA), London, 8-9 June 2015, 1-6. https://doi.org/10.1109/CyberSA.2015.7166111

[10] Stanger, N., Alnaghaimshi, N. and Pearson, E. (2017) How Do Saudi Youth Engage with Social Media? First Monday, 22, 1-20. https://doi.org/10.5210/fm.v22i5.7102

[11] Alanzi, T.M., Alobrah, A., Alhumaidi, R. and Alorai, S. (2018) Evaluation of the Snapchat Mobile Social Networking Application for Breast Cancer Awareness among Saudi Students in the Dammam Region of the Kingdom of Saudi Arabia. Breast Cancer (Dove Med Press), 10, 113-119.

https://doi.org/10.2147/BCTT.S166135

[12] DeLegge, A. and Wangler, H. (2017) Is This the End of Facebook? A Mathematical Analysis. Applied Mathematics and Computation, 305, 364-380. https://doi.org/10.1016/j.amc.2017.02.014

[13] Edelstein-Keshet, L. (2005) Mathematical Model in Biology. Society for Industrial and Applied Mathematics. https://doi.org/10.1137/1.9780898719147

[14] (2017) Annual Year Book. https://www.stats.gov.sa/en/46 